$x-612-65-79$

\title{
EXTENSION OF THE PHOTOSPHERIC MAGNETIC FIELD INTO INTERPLANETARY SPACE
}

\author{
By \\ Norman F. Ness \\ Goddard Space Flight Center \\ Greenbelt, Maryland \\ and \\ John M. Wilcox \\ Space Sciences Laboratory \\ University of California \\ Berkeley, California
}

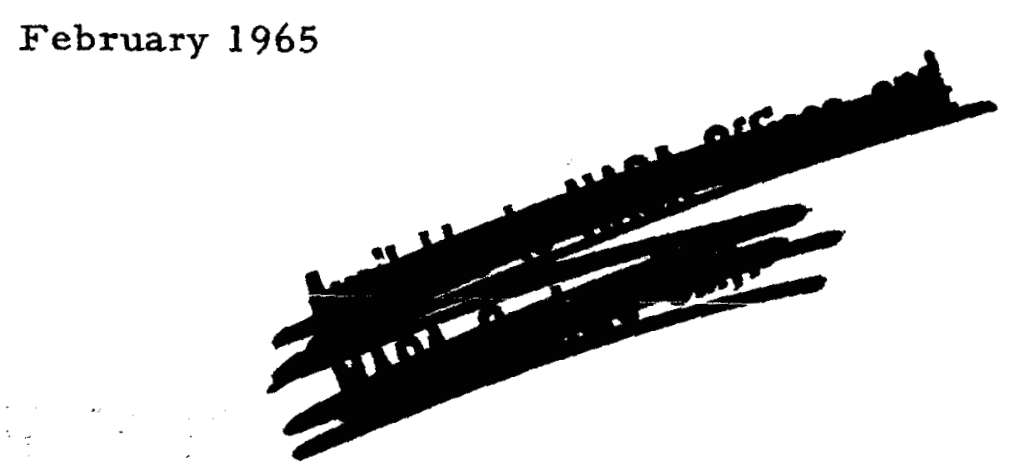


Extension of the Photospheric Magnetic Field

into Interplanetary Space

\author{
Norman F. Ness \\ NASA-Goddard Space Flight Center \\ Greenbelt, Maryland
}

and

John M. Wilcox

Space Sciences Laboratory

University of California

Berkeley, California

22260

Abstract: Observations of the nearby interplanetary magnetic field by the magnetometer experiment on the artificial Earth satellite IMP-1 have been compared with observations of the photospheric magnetic field obtained with the solar magnetograph at the Mt. Wilson Observatory. A good correlation has been found between the large scale pattern of the direction (into or out of the sun) of the photospheric field and the pattern of the direction (toward or away from the sun) of the nearby interplanetary field. This substantiates the theoretical model of the transport of solar magnetic fields into interplanetary space by the magnetohydrodynamic expansion of the solar corona.<smiles>CC1CC1CC1CC1</smiles> 
CONTENTS

$\underline{\text { Page }}$

Introduction $\ldots \ldots \ldots \ldots \ldots \ldots \ldots \ldots \ldots \ldots \ldots \ldots \ldots \ldots \ldots$

Photospheric Magnetic Field ....................

Nearby Interplanetary Magnetic Field .............. 5

Comparison of the Photospheric Field

and the Interplanetary Field ..................

Interplanetary Magnetic Field Component

Perpendicular to the Ecliptic ..................

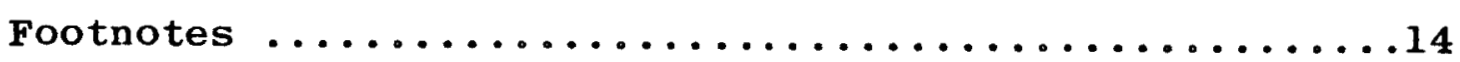

Acknowledgements $\ldots \ldots \ldots \ldots \ldots \ldots \ldots \ldots \ldots \ldots \ldots \ldots \ldots \ldots \ldots \ldots$

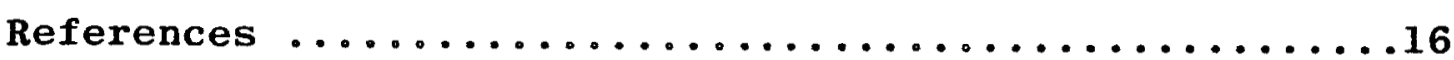

Figure Captions $\ldots \ldots \ldots \ldots \ldots \ldots \ldots \ldots \ldots \ldots \ldots \ldots \ldots \ldots \ldots \ldots$

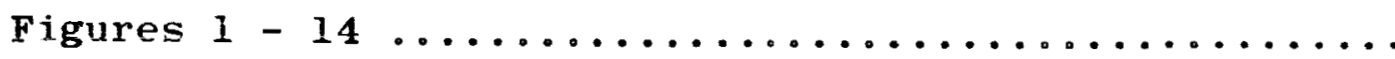


Extension of the Photospheric Magnetic Field

into Interplanetary space

Introduction

Satellite measurements show that the solar plasma flux (solar wind) always has a magnetic field associated with it. Near 1 AU the magnetic energy density is about two orders of magnitude smaller than the kinetic energy density associated with the streaming motion of the plasma. Present experimental data are limited but indicate that the magnetic energy density may be comparable with the thermal energy density of the plasma. Under these conditions the magnetic field lines are "frozen" into the plasma and transported along with it. It is possible that the pattern of the photospheric magnetic field is maintained up to the height at which the solar wind begins to form, and that thereafter this pattern is frozen into the solar wind and transported out to 1 AU where it can be examined with satellite magnetometers. Although during quiet conditions the transport of magnetic lines may be rather passive at large distances from the Sun, this would presumably not be the case close to the Sun where the magnetic energy density is larger than the plasma energy density so that the magnetic fields can influence the plasma flow, either by directly exerting forces on the plasma or by influencing the coronal heating and thus the flux of the solar wind. An extreme alternative to the above considerations would be that somewhere between the photosphere and the position of the satellite, turbulence and 
and disruptive effects would be sufficient to completely destroy the pattern of the photospheric magnetic field.

In this paper the influence of photospheric magnetic field regions whose size corresponds to a few day's solar rotation is investigated. Future work will consider the situation with regard to solar structures that are smaller in time and extent. 


\section{PHOTOSPHERIC MAGNETIC FIELD}

The observations of the photospheric magnetic field made with the solar magnetograph (Babcock, 1953) at the ut. Wilson Observatory were kindly made available to us by Dr. Robert Howard. From the individual solar magnetograms obtained each day (weather permitting) synoptic charts are prepared (see Figure 1). The Cx portion of each daily solar magnetogram is preferentially used in preparing the synoptic chart, although occasionally regions removed from $\mathrm{CM}$ are used to fill in for days when the daily magnetograms cannot be obtained. The lowest contour represents a field of 2 gauss (averaged over an area on the sun of $4 \times 10^{8} \mathrm{~km}^{2}$ ), and ine magnetograph saturates at about 70 gauss. Thus the synoptic charts display regions of considerable spatial extent containing relatively weak magnetic fields. A comparison of synoptic charts for two successive solar rotations shows a large degree of similarity, indicating that many of the features have a lifetime that is at least comparable with the solar rotation period. The large but localized magnetic fields of sunspots do not appear on these charts, and discrete events such as flares also are not recorded.

For comparison with the satellite observations of the nearby interplanetary magnetic field, the synoptic charts of the photospheric magnetic field are analysed as follows. A strip of width $10^{\circ}$ in latitude centered about a given latitude is divided into segments with a longitudinal width 
corresponding to the solar rotation in twelve hours. A magnetic field direction (into or out of the sun) is assigned to each segment only if at least 70 or 80 percent of the area within the segment has a field sense in this direction. If an area does not fall within any contour level but is nevertheless closely surrounded by contours all of the same field direction, then the area is assumed to have a weak field in this direction. The relatively weak solar magnetic fields existing during this time of minimum activity make this procedure necessary. Thus the photospheric field direction at a given latitude is described by a series in which time is divided into twelve hour intervals and each interval is labeled either +1 (field predominantly directed out of the sun), -1 (field predominantly directed into the sun), or 0 (field direction ambiguous or no observations available). There are gaps in the photospheric data for about 25 days caused by weather conditions at Mt. Wilson. This process is repeated at $5^{\circ}$ intervals in latitude from $25^{\circ} \mathrm{N}$ to $25^{\circ} \mathrm{S}$ of the center of the visible disk. The coordinate system is centered in latitude about the center of the visible disk rather than about the solar equator because the disk center would be the source of the solar wind that flows past the Earth if the solar wind velocity were entirely radial. 


\section{NEARBY INTERPLANETARY MAGNETIC FIELD}

The satellite IMP-1 (Interplanetary Monitoring Platform) was launched on Nov. 27, 1963 and provided accurate measurements (Ness, Scearce and Seek, 1964; Ness and Wilcox, 1964) of the relatively undisturbed interplanetary field until the middle of February 1964. The highly eccentric orbit has an apogee of 31.7 Earth radii and a period of 93.5 hours. The Sun-Earth-apogee angle was initially $25^{\circ}$ west of the Sun and increased approximately $1^{\circ}$ per day because of the Earth's motion about the sun, as shown in Figure 2. The interaction of line soiar wind with the geomagnetic field disturbs the interplanetary medium in the immediate vicinity of the earth and spatially confines the Earth's field. Measurements of the relatively undisturbed interplanetary medium by IMP-1 were restricted to times centered about apogee when the satellite was outside of the interaction region. During each of the early orbits interplanetary medium measurements could be obtained for about three days. This interval gradually decreased (see Figure 2) until in the middle of February IMP-1 was entirely within the interaction region.

The two fluxgate magnetometers on IMP-1 gave a measurement of the vector magnetic field once every 20.48 seconds. The average magnitude of the field was $5.0 \gamma\left(1 \gamma=10^{-5}\right.$ gauss) with an uncertainty of $1 / 4 \gamma$. Details about the magnetometers 
and telemetry sequence are given in Ness, Scearce and Seek (1964). The observations are consistent with Parker's (1958) model of an interplanetary field of solar origin in which the effects of a radial solar wind velocity, the highly ionized solar plasma, and the solar rotation combine to produce magnetic lines of force twisted in the plane of the ecliptic in the form of an Archimedes spiral. Figure 3 shows the theoretical interplanetary magnetic field streaming angle as a function of the solar wind velocity that is predicted by this model. Figure 4 shows the measured distribution of the interplanetary field direction. The initial twenty second measurements are combined into 5.46 minute averages, and the number of such measurements per unit solid angle is shown for the field component in the ecliptic plane and for the component normal to the ecliptic plane. An isotropic distribution of the same number of vectors would produce the dashed circles shown in Figure 4. The observed field component in the ecliptic plane is seen to be preferentially in the directions predicted by Parker's model. From inspection of the distribution of the direction of the field component normal to the ecliptic plane one can see that the vector field is predominantly parallel (rather than perpendicular) to the ecliptic plane. A few remarks on this component are included at the end of this note.

For comparison with the solar magnetograph observations of the photospheric magnetic field the satellite measurements 
are analysed in a similar fashion. The direction angle in the ecliptic plane is divided into $20^{\circ}$ sectors. Each three hour interval of time is assigned to the sector which contains the largest number of the 5.46 minute average directions within that interval. As shown in Figure 4, the sectors from $90^{\circ}$ to $230^{\circ}$ East of the Earth-Sun direction are assumed to represent a magnetic field directed predominantly away from the Sun (along the spiral streaming angle) and are labeled +1. Similarly the sectors from $270^{\circ}$ to $50^{\circ}$ East of the Earth-Sun direction are assumed to represent a field directed predominantly toward the Sun and are labeled -1 . The shaded sectors are omitted from this analysis. Four 3 hour intervals are combined into a 12 hour interval which is labeled +1 or -1 only if at least three of the 3 hour intervals have the same sign. Thus a time series is constructed on a 12 hour interval which represents the large scale direction of the interplanetary field in the sense of being either toward or away from the Sun.

A solar source for the interplanetary magnetic field should produce a recurrence tendency in the satellite observations corresponding to one solar rotation period. An autocorrelation $^{1}$ of the 12 hour IMP time series displays a prominent peak at a 1 ag of about 27 days with an amplitude of $0.55 \pm 0.5$. It was observed that the data of the first eight days (including the magnetic storm of December 2) 
produced most of the exceptions to the recurrence tendency, and that if this data were omitted the 27 day peak amplitude increased to $0.85 \pm 0.10$, as shown in Figure 5. Therefore the data of the first eight days are omitted from the further analysis in order to study a time in which the recurrence tendency is very prominent. 


\section{COMPAR ISON OF THE PHOTOSPHERIC FIELD}

AND THE INTERPLANETARY FIELD

A cross-correlation between the IMP-1 twelve hour time series and the twelve hour series representing the photospheric field at a given latitude is then computed. The results for solar latitudes from $25^{\circ} \mathrm{N}$ to $25^{\circ} \mathrm{S}$ of the center of the visible disk are shown in Figures 6-11. The abscissa is the time lag from CM passage of the photospheric field area to the time the field was measured by IMP-1. The prominent positive peak at a lag of approximately $41 / 2$ days is common to all of the latitudes examined. A peak in the correlation at a lag of $41 / 2 \pm 1 / 2$ days would correspond to an average radial solar wind velocity of $385 \pm 45 \mathrm{~km} / \mathrm{sec}$. The average radial solar wind velocity from Nov. 27, 1963 to Feb. 11, 1964 measured by the MIT plasma dectector experiment on IMP-1 (Bridge, Egidi, Lazarus, Lyon and Jacobson, 1964) was $319 \mathrm{~km} / \mathrm{sec}$. The significance of this difference is not yet clear. If it persists in future observations it may help to determine the conditions in the region within a few solar radii of the sun in which the magnetic energy density is greater than the plasma energy density. It may suggest that the magnetic 1 ines in this region are preferentially tipped forward in the sense of the solar rotation. 
Inspection of Figure 1 shows that the pattern of the photospheric magnetic field at this time is dominated by regions of a single polarity (into or out of the Sun) with an extent in both latitude and longitude covering an appreciable fraction of the solar disk. Note that a region often extends on both sides of the solar equator. Because of the predominance of these large regions it is reasonable that the cross-correlation curves shown in Figures 6-11 should display similar peak correlations. This limits the precision with which the latitude or latitudes of the solar source of the interplanetary field can be determined by this method. Therefore the differential rotation of the photospheric magnetic field was investigated. The recurrence period of the nearby interplanetary magnetic field (Figure 5) should be the same as the average rotation period of the latitudes of the solar sources. An autocorrelation of the twelve hour photospheric field data described above is computed for each latitude. The results for the center of the visible disk and for $25^{\circ} \mathrm{N}$ thereof are displayed in Figure 12. The rotation period of the photospheric field as a function of latitude as determined in this manner ${ }^{2}$ is shown in Figure 13. The solid line is from the observations of long-lived sunspots by Newton and Nunn (1951). The uncertainties are estimated by dividing the data sample into three parts and performing the autocorrelation on each part. Data for approximately three 
solar rotations is available; the first rotation is correlated with the second and with the third, and the second rotation is correlated with the third.

The large arrow at the left of Figure 13 indicates the recurrence period of the IMP-I magnetic field as computed from the autocorrelation shown in Figure 5. The uncertainties indicated by the small arrows are computed in the manner described above. The comparison in Figure 13 of the interplanetary field recurrence period with the differentia? -ntation nf th- photospheric field suggests that the latitude of the photospheric source of the interplanetary field was within 10 or 15 degrees of the center of the visible disk (or of the equator; the present analysis cannot make a distinction between the two).

These results indicate that during three solar rotations near the minimum of the solar cycle, some of the magnetic field lines passing through the photosphere near the center of the visible disk tended to be transported out by the solar wind plasma to become part of the nearby interplanetary magnetic field. The best correlation was obtained when the photospheric field was in the same direction throughout an area corresponding to at least two or three days rotation. These conclusions are consistent with the model suggested by Ahluwalia and Dassler (1962) in which the sense of the interplanetary magnetic field sectors is related to the sense of photospheric magnetic field regions. 


\section{INTERPLANETARY MAGNETIC FIELD COMPQNENT \\ PERPENDICULAR TO THE ECLIPTIC}

\section{A few preliminary remarks are addressed to the question} of the cause of the net southward component of the nearby interplanetary magnetic field shown in the right hand side of Figure 4. The measured field had a southward component during $2 / 3$ of the three hour intervals of the IMP-1 observations, and the average component perpendicular to the ecliptic during this time was about $0.5-1.0 \mathrm{y}$ in the southward direction. Photospheric magnetic polarities at tho timo of these IMP-1 nhservations are schematically indicated in Figure 14. The bipolar magnetic regions BMR in each hemisphere have the indicated polarities. The following portions drift slowly poleward and produce the polar magnetic fields while the preceding portions drift towards the equator and tend to merge (Babcock, 1961). The magnetic 1 ines connecting the two portions of a BMR have a northward component in both hemispheres, as shown in Figure 14. However magnetic lines from a preceding portion of a BMR in the northern hemisphere connecting to a preceding portion of a BMR in the southern hemisphere would have the required southward component. This feature of the photospheric field structure could pussibly be related to the southward component of the interplanetary field. Unipolar magnetic regions (UMR) usually 
form from the following portions of BMRs and thus have the polarities indicated in Figure 14. Magnetic lines from the low latitude side of a UMR in either hemisphere would have a northward component. Finally the northern polar region has a net field directed into the Sun while the net field in the southern polar region is nearly zero (Howard, 1963). Magnetic lines approaching the northern polar region would also have a northward component. Future observations with the solar magnetograph of the transverse component of the photospheric magnetic field may help to resolve this question. 


\section{FOOTNOTES}

1. In computing the "correlations" described in this note a weight of +1 was assigned to a field directed out of or away from the Sun, and vice versa for -1 . Each individual set of data did not add up exactiy to zero. If the data sample were enough that each set of data would sum to zero then this computation would yield a formal correlation. The error is estimated by dividing the data sample into three parts and repeating the computation on each portion.

2. The differential rotation of the photospheric magnetic field observed over several years will be reported in a future publication. 


\section{Acknowledgements}

We thank C. S. Scearce and J。B. Seek for their important participation in the IMP-1 magnetic field experiment. We thank Dr. Robert Howard for making the solar magnetograms available, and Dr. Howard and Dr. V. Bumba for several valuable discussions. One of us (JMW) thanks the director of the Mt. Wilson Observatory for guest investigator privileges at the observatory. 


\section{REFERENCES}

Ahluwalia, H. S. and Dessler, A. J. 1962, Planet. Space Sci. $\underline{9}, 195$.

Babcock, H. W. 1953 , Ap. J. 118, 387 .

Babcock, H. W. 1953, Ap. J. 133, 572 .

Bridge, H., Egidi, A., Lazarus, A., Lyon, E. and Jacobson, L. 1964, private communication.

Howard, R. 1963, "Proceedings of the IAU Symposium on Solar and Stellar Magnetism", (to be published).

Ness, N. F., Scearce, C. S. and Seek, J. B. 1964, J. Geoph ys. Res. 69,3531 .

Ness, N.F. and Wilcox, J. M. 1964, Phys. Rev. Lett. 13, 461 .

Newton, H.W. and Nunn, M. L. 1951, M. N. 111, 413 .

Parker, E。N. 1958, Ap. J. 128, 664 . 
FIGURE CAPTIONS

Figure 1. Synoptic chart of the photospheric magnetic field. Solid contours represent a field directed out of the Sun and dashed contours represent a field directed into the Sun. Contour levels are 2, 4, 8, 12 and 25 gauss.

Figure 2. Relation of IMP-1 orbit trajectories to the shock wave and magnetopause. Measurements of the relatively undisturbed interplanetary medium are possible only when the satellite is outside of the shock wave.

Figure 3. Theoretical interplanetary magnetic field streaming ang? $:$.

Figure 4. Measured distribution of interplanetary field directions 。

Figure 5. Autocorrelation of the measured interplanetary field directions. Data for the first 8 days are omitted.

Figure 6. Cross correlation of nearby interplanetary magnetic field direction and photospheric magnetic field direction for solar latitudes $25^{\circ} \mathrm{N}$ and $20^{\circ} \mathrm{N}$ of the center of the visible disk.

Figure 7. Same as Figure 6 for latitudes $15^{\circ} \mathrm{N}$ and $10^{\circ} \mathrm{N}$.

Figure 8. Same as Figure 6 for latitudes $5 \mathrm{~N}$ and the Center of the visible disk.

Figure 9. Same as Figure 6 for latitudes $5^{\circ} \mathrm{S}$ and $10^{\circ} \mathrm{S}$. 
Figure 10. Same as Figure 6 for latitudes $15^{\circ} \mathrm{S}$ and $20^{\circ} \mathrm{S}$. Figure 11. Same as Figure 6 for latitude $25^{\circ} \mathrm{S}$.

Figure 12. Autocorrelation of the photospheric magnetic field direction for latitudes at the center of the $\mathrm{visible}$ disk and $25^{\circ} \mathrm{N}$ thereof. The increase in period caused by differential rotation is quite apparent.

Figure 13. Rotation period of the photospheric magnetic field as a function of latitude, as determined from autocorrelation analysis as shown in Figure 12. The solid line is the rotation period determined by Newton and Nunn from analysis of long-lived sunspots. The arrows at left indicate the recurrence period of nearby interplanetary field and the uncertainty thereof.

Figure 14. Schematic diagram of some photospheric magnetic field polarities at the time of these observations. BMR is bipolar magnetic region and UMR is unipolar magnetic region. A plus sign indicates a field directed out of the sun and a minus sign indicates a field directed into the Sun. Directions of possible magnetic lines connecting various regions are indicated by the heavy arrows. 


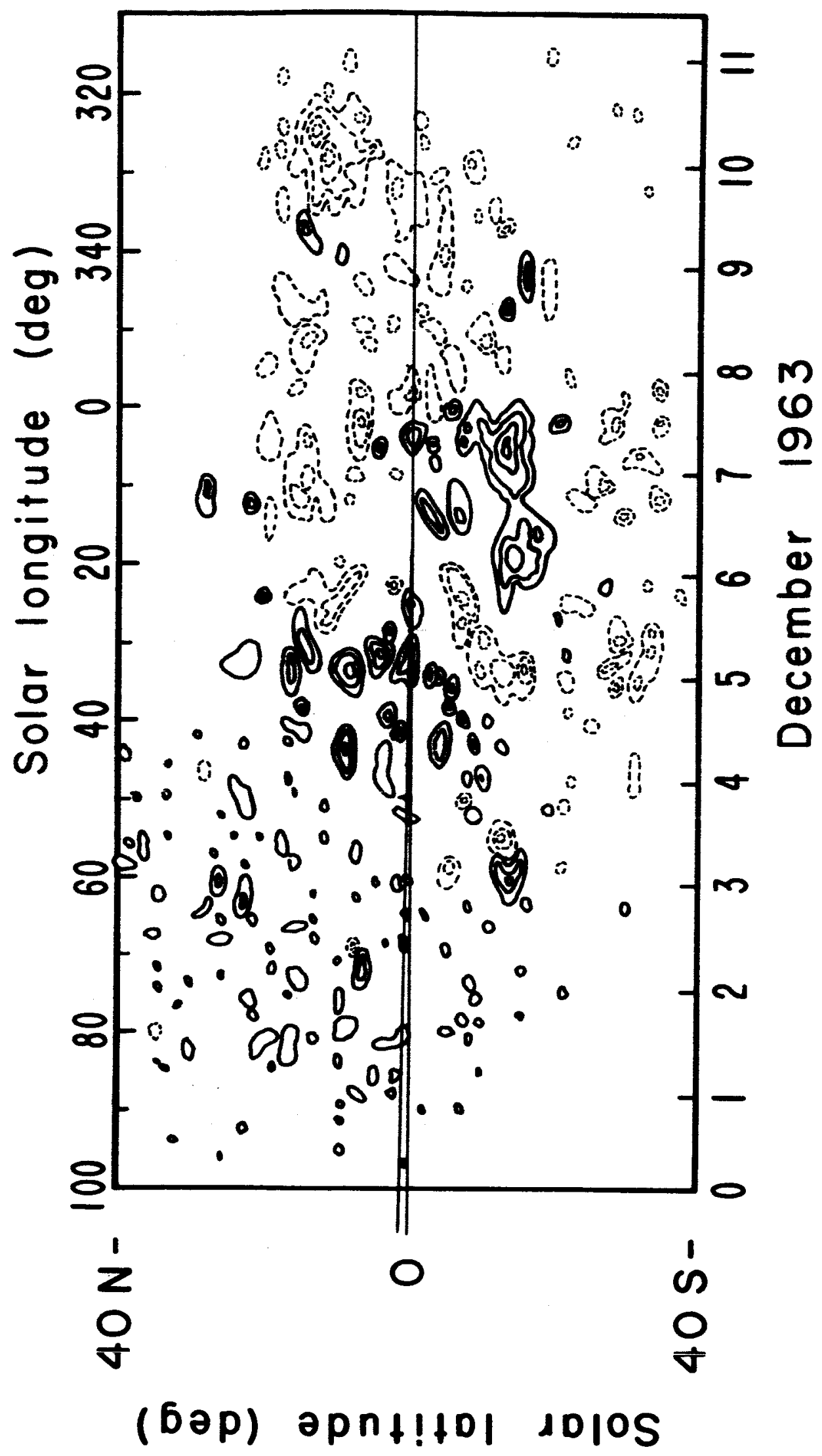

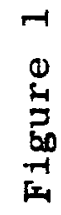




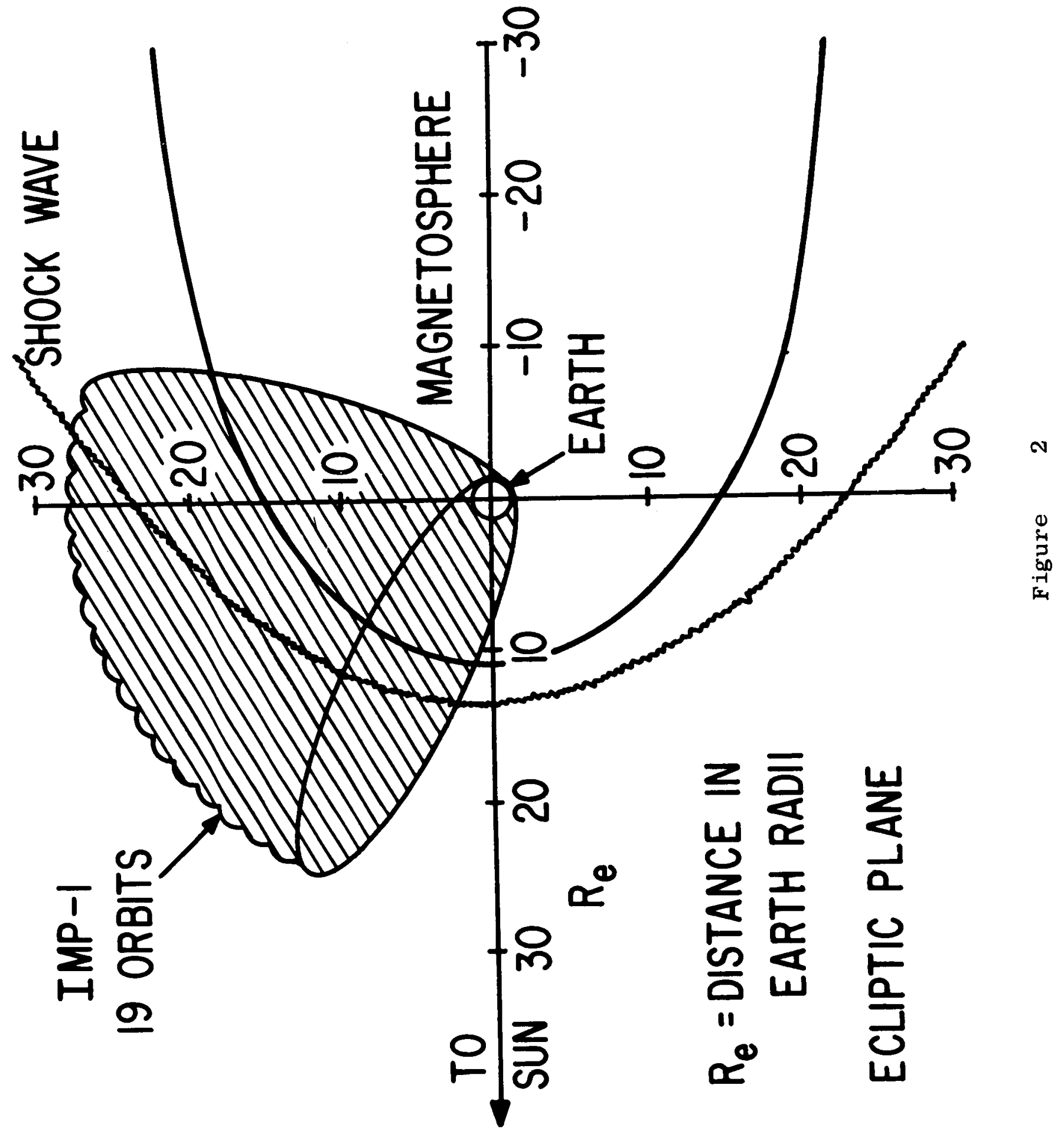




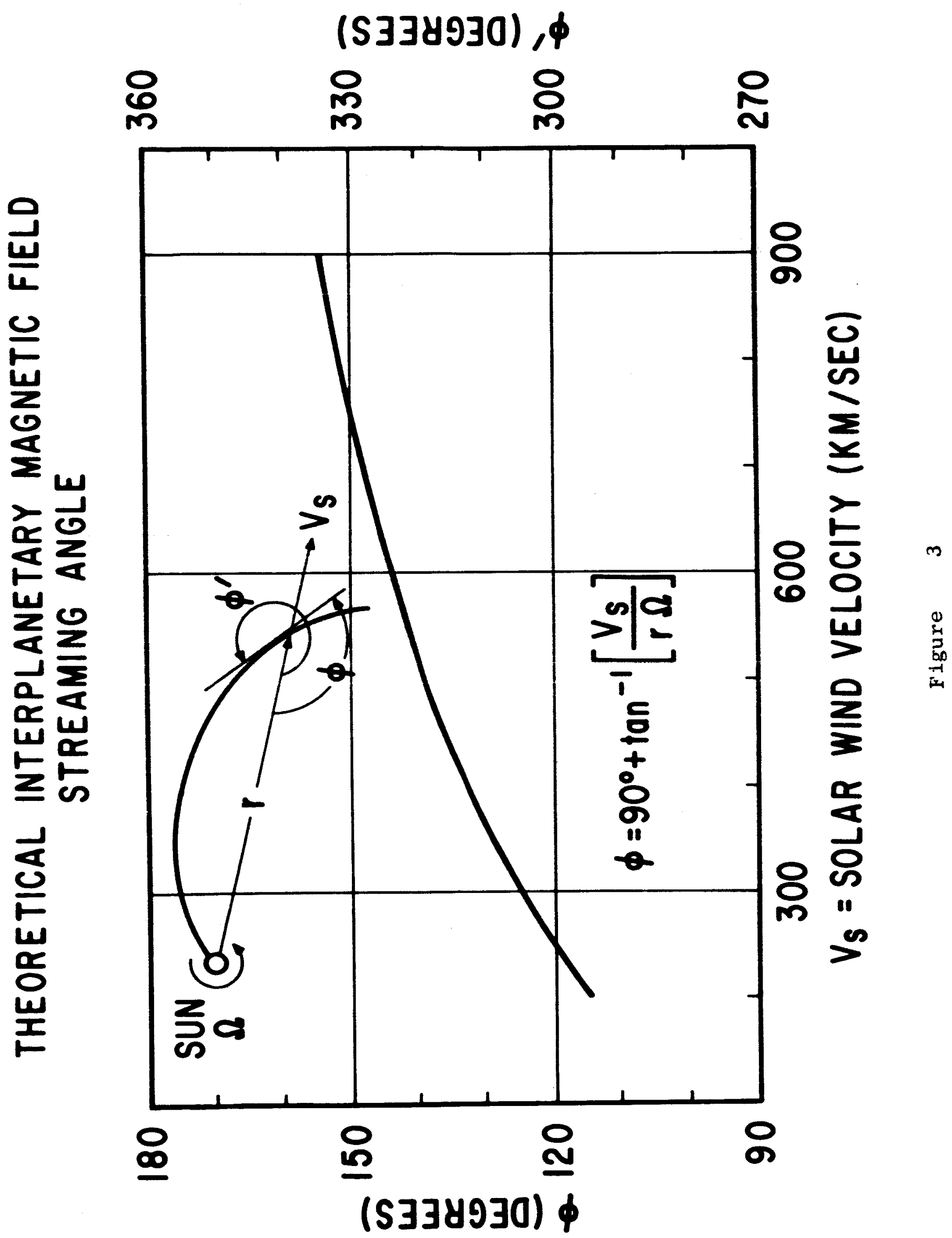




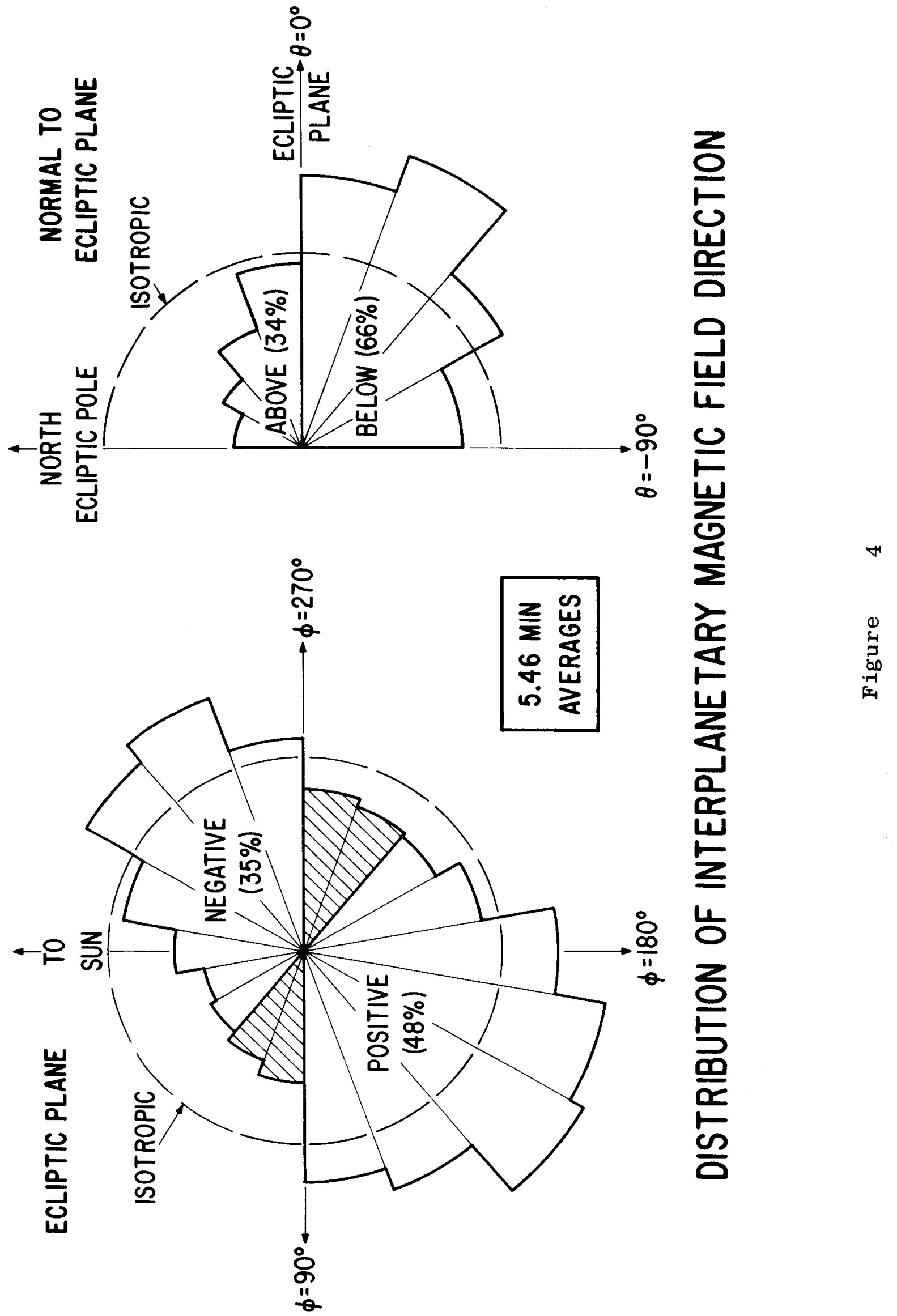




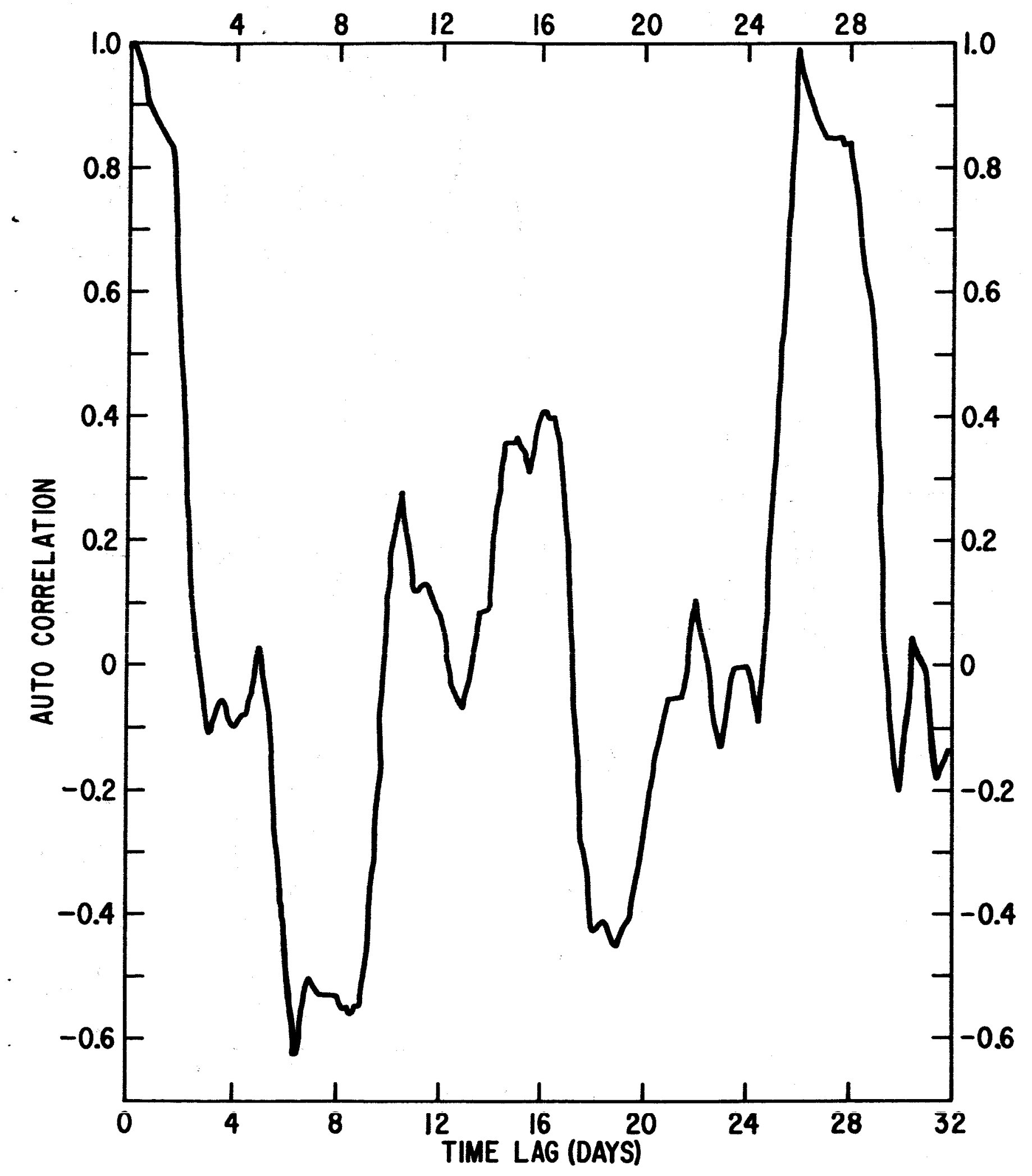

Figure 5 


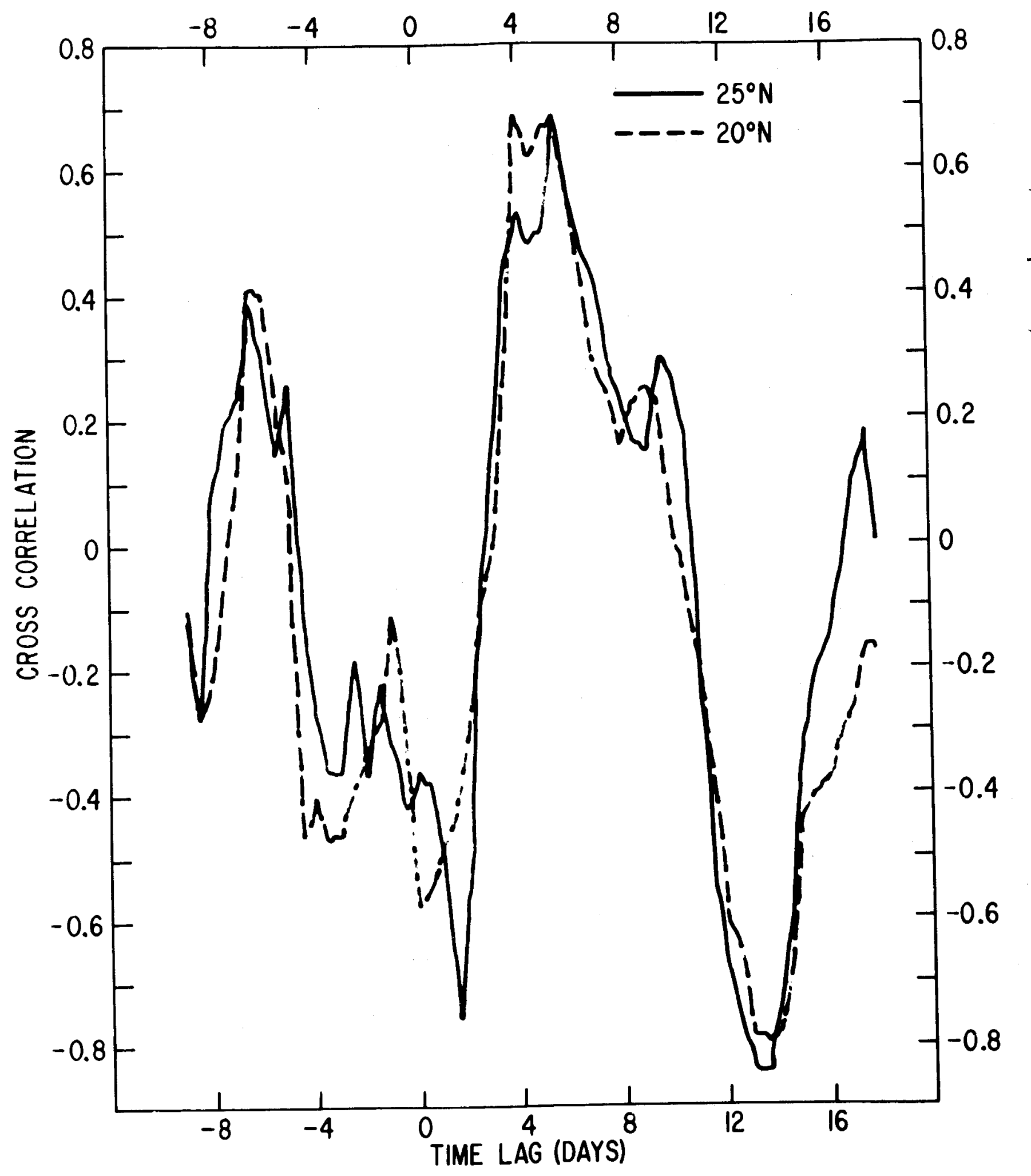

Figure 6 


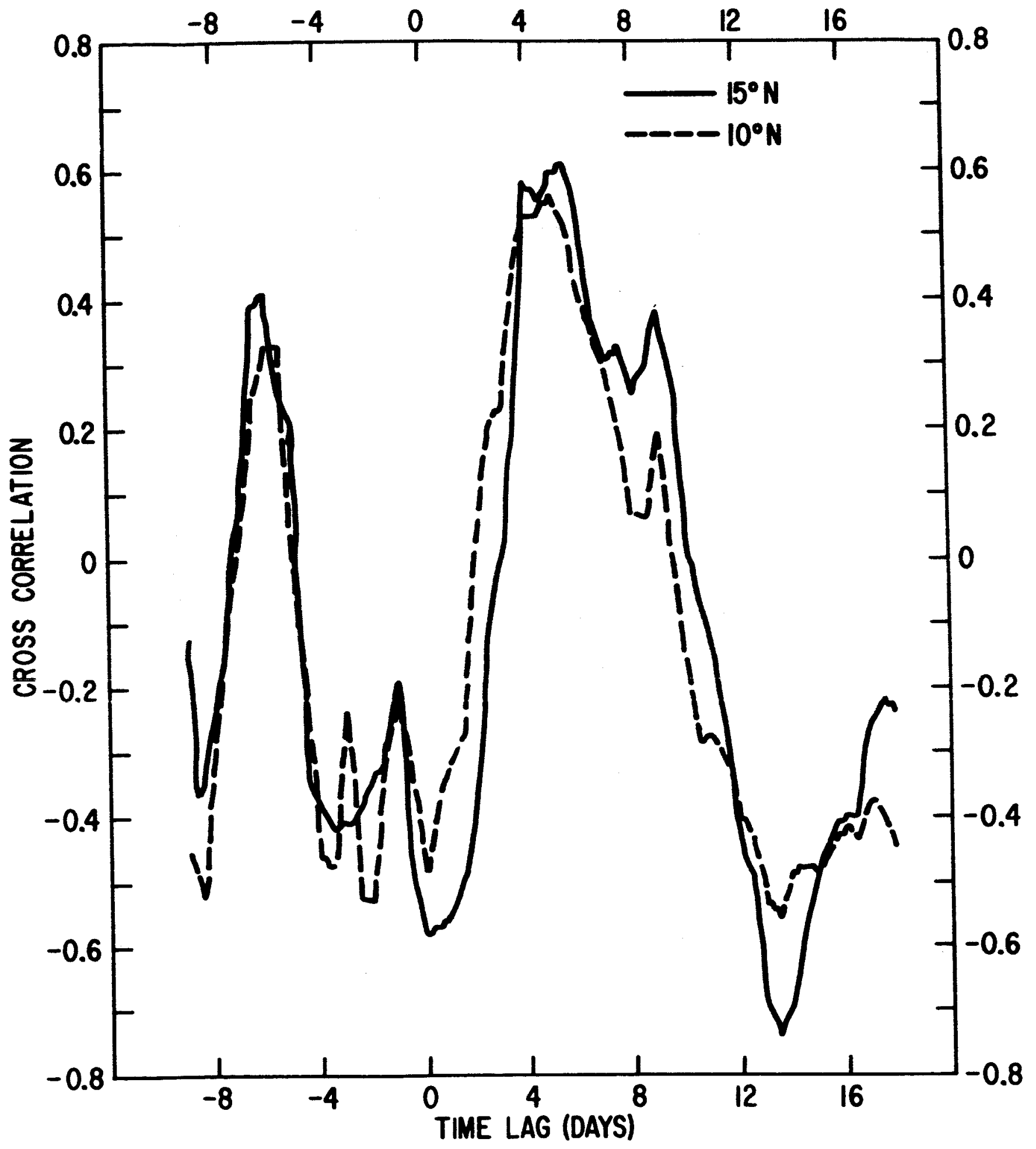

Figure 7 


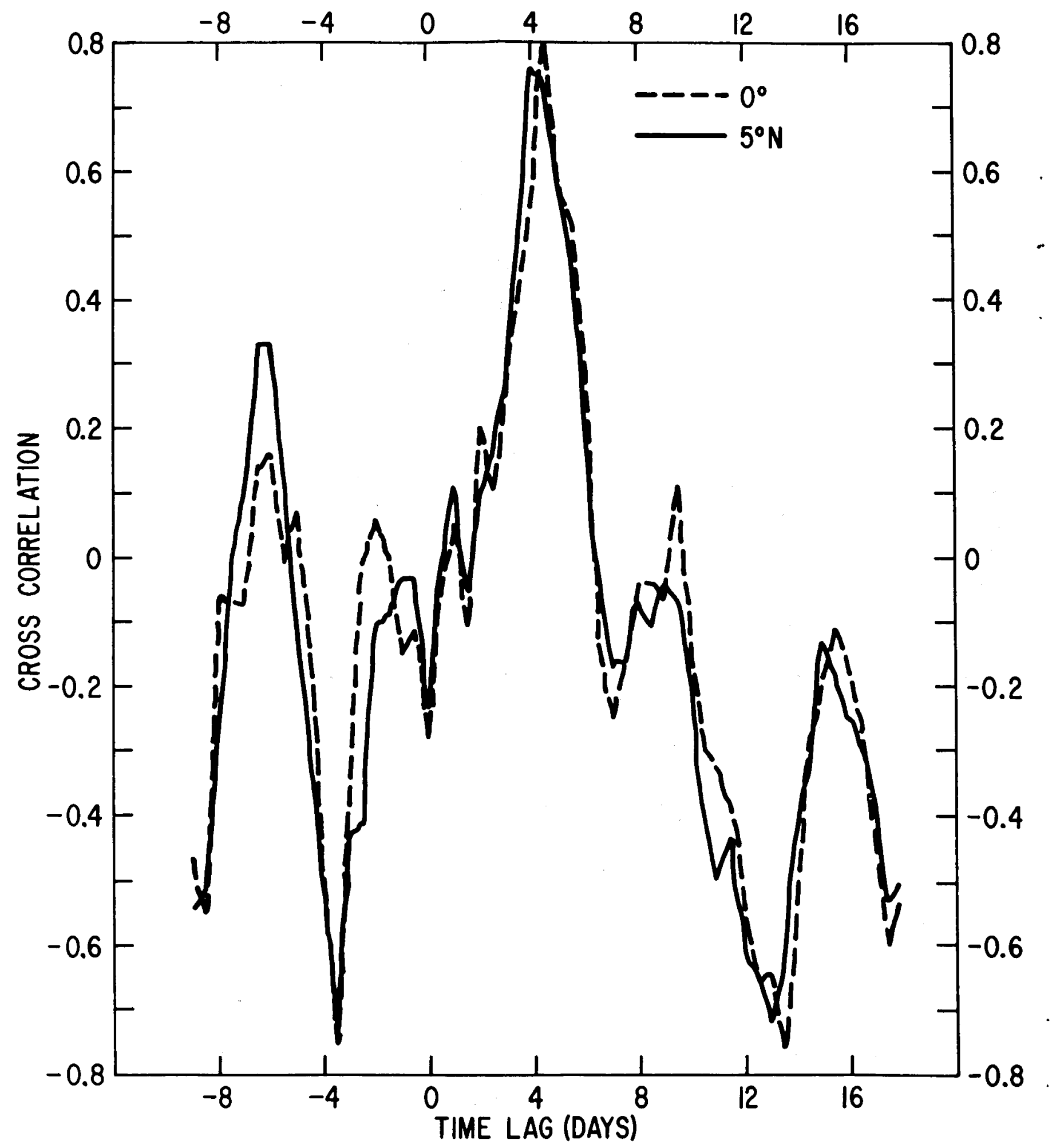

Figure $\quad 8$ 


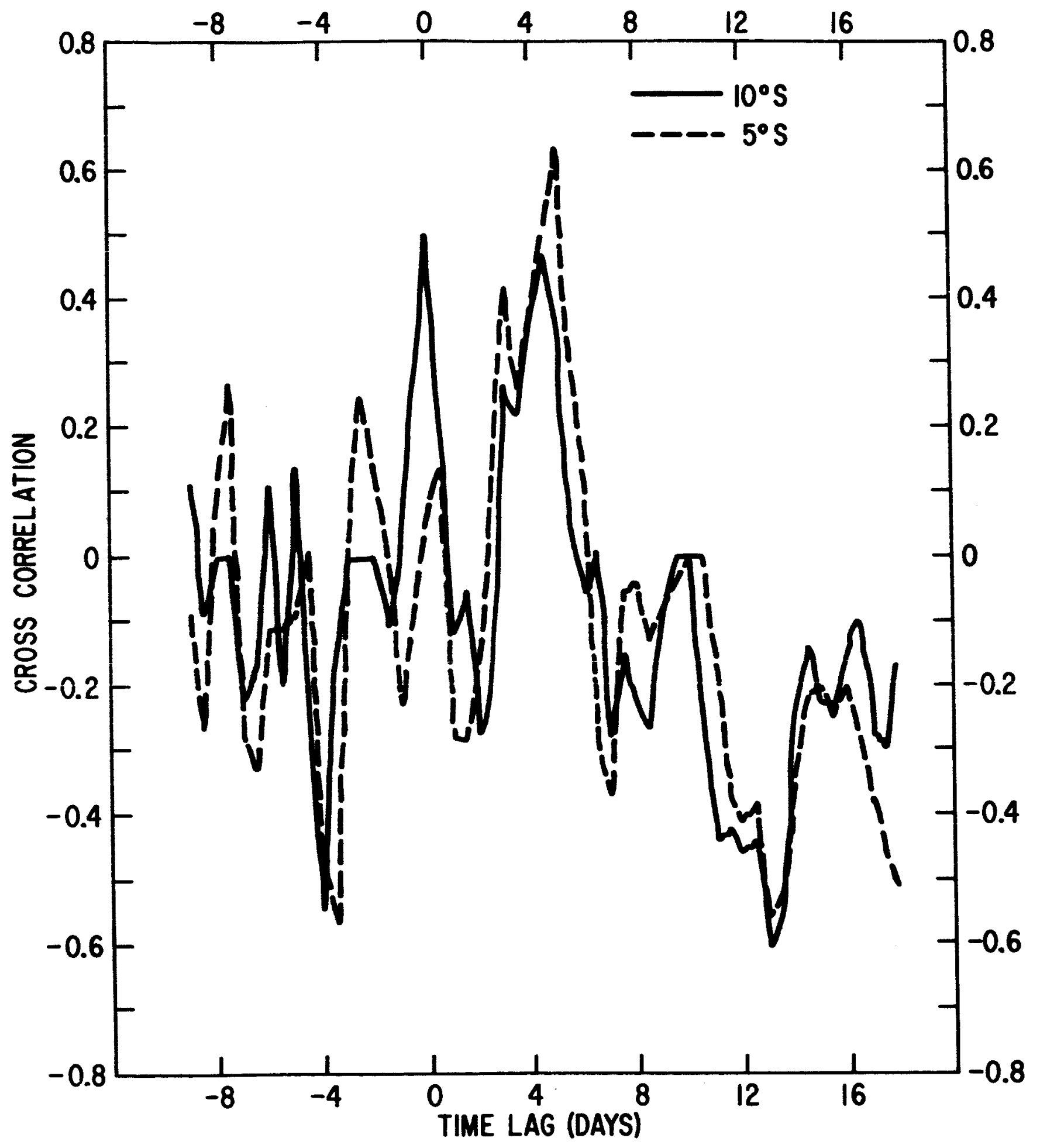

Figure 9 


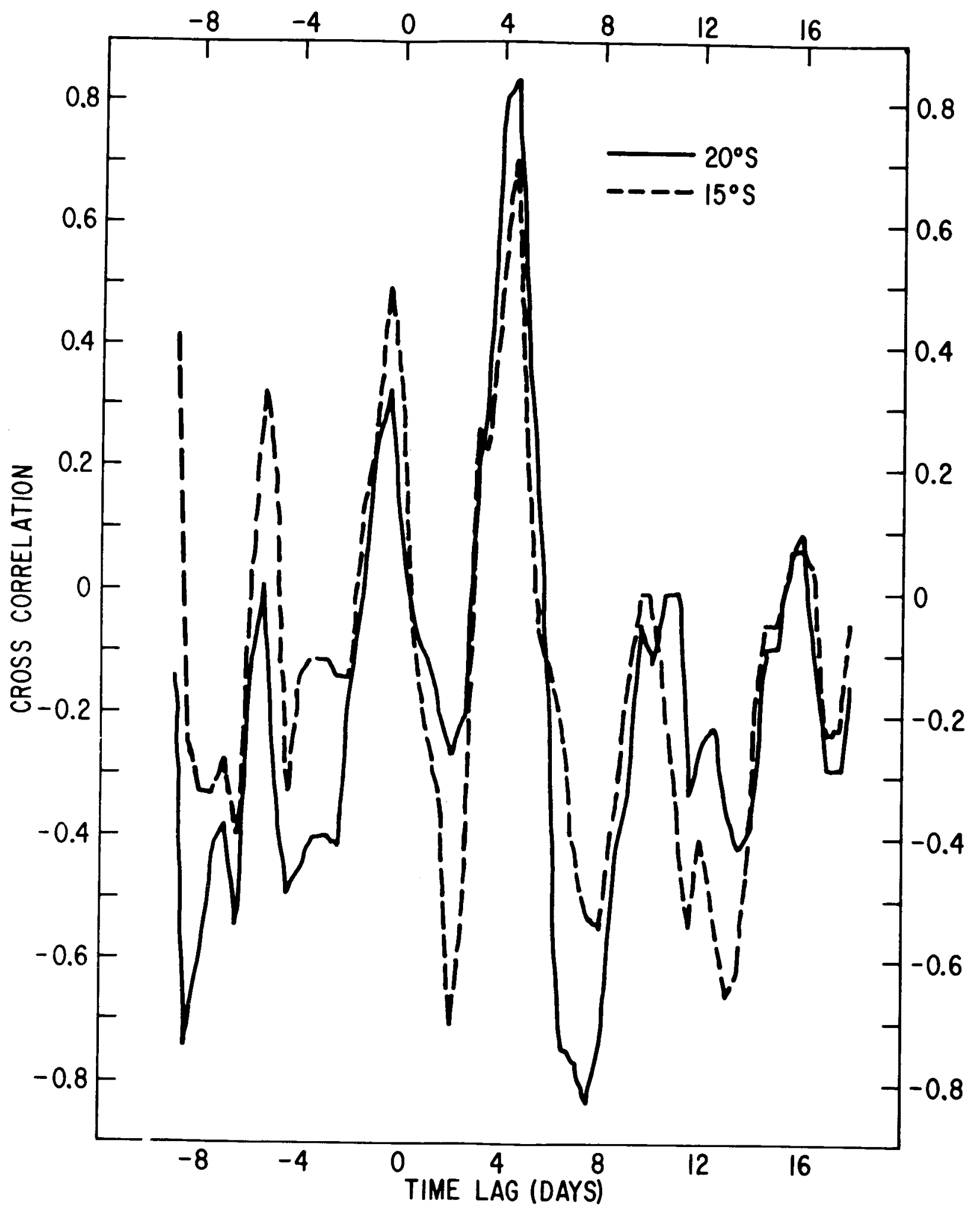

Figure 10 


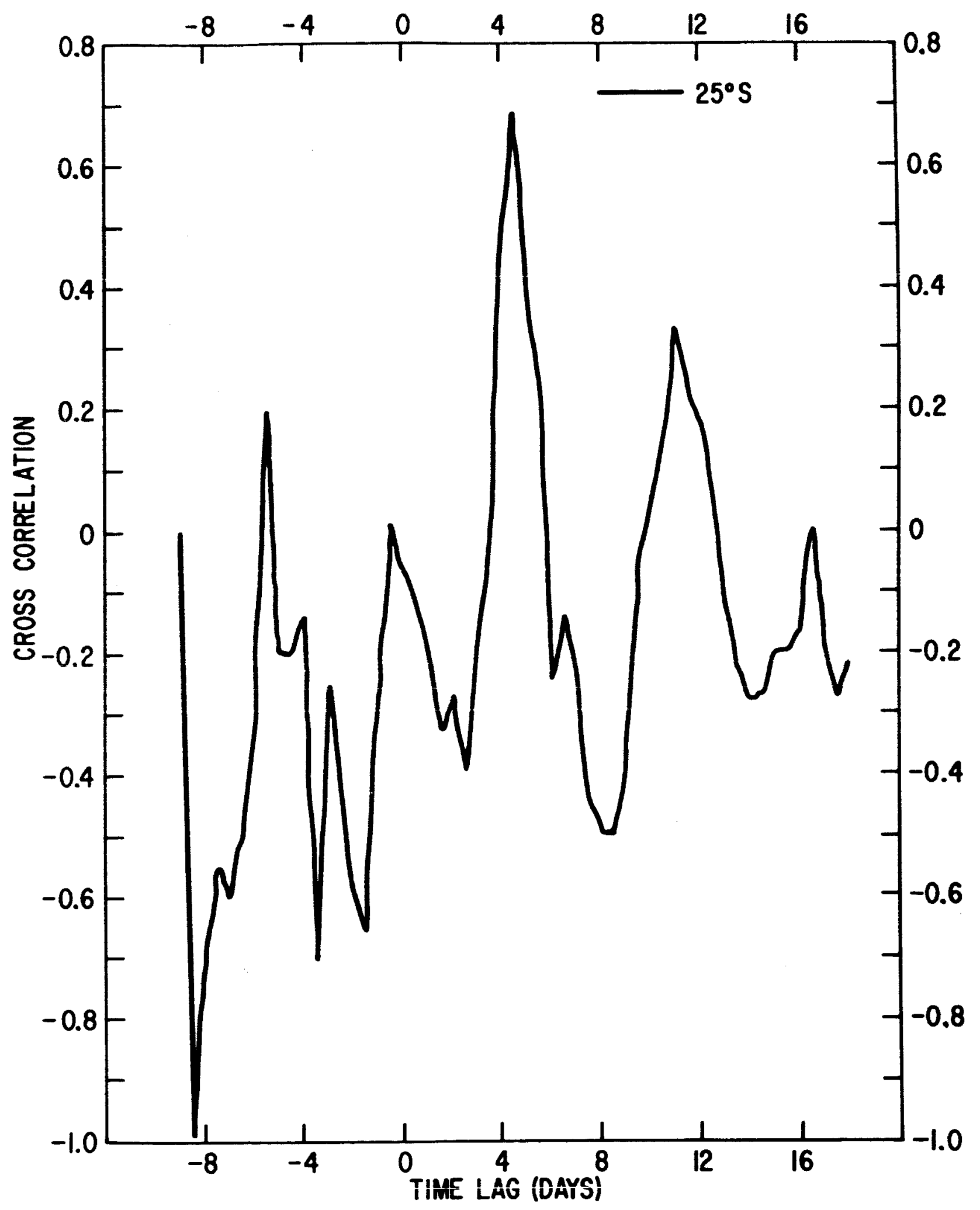

Figure $\quad 11$ 


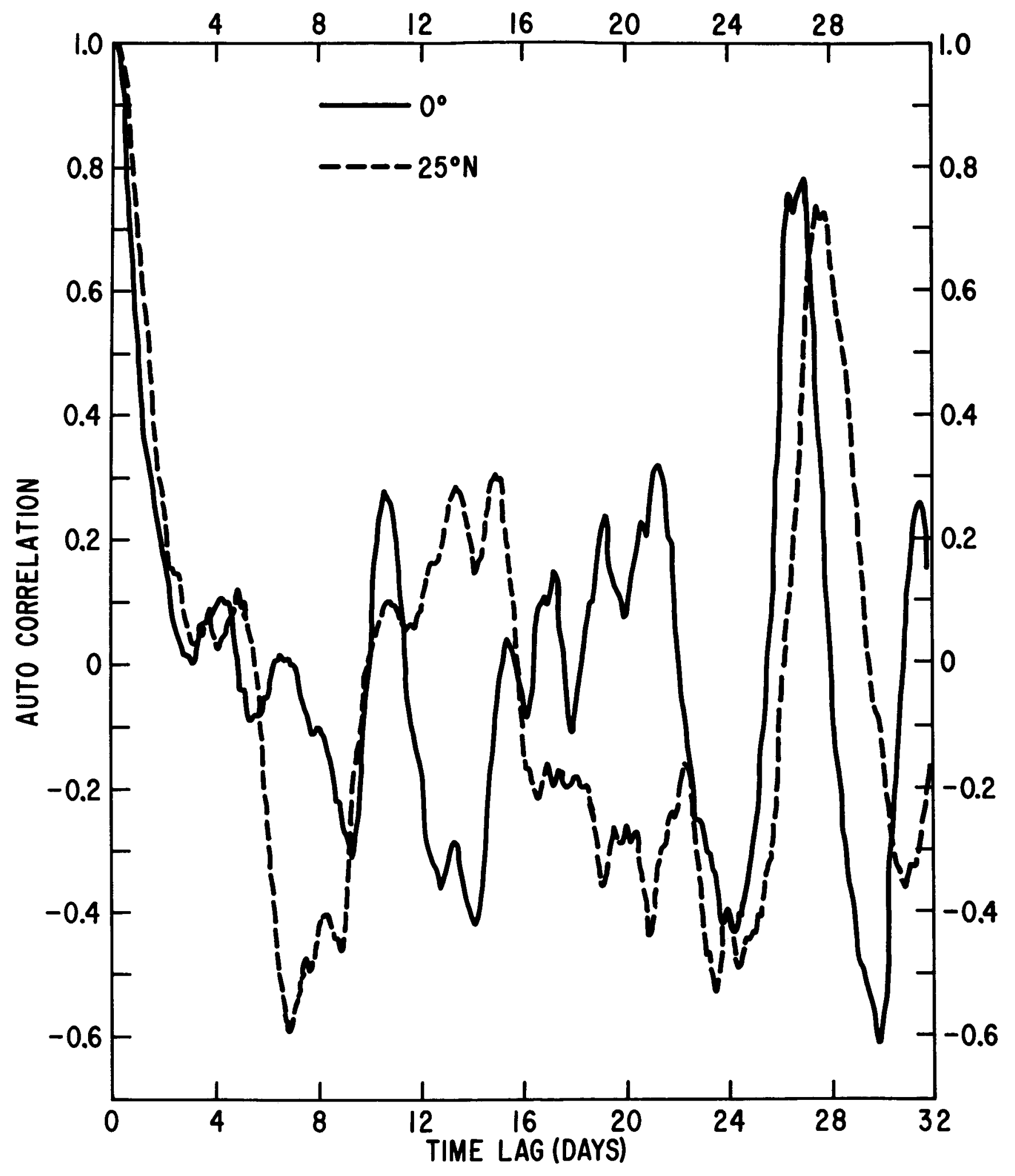

Figure $\quad 12$ 


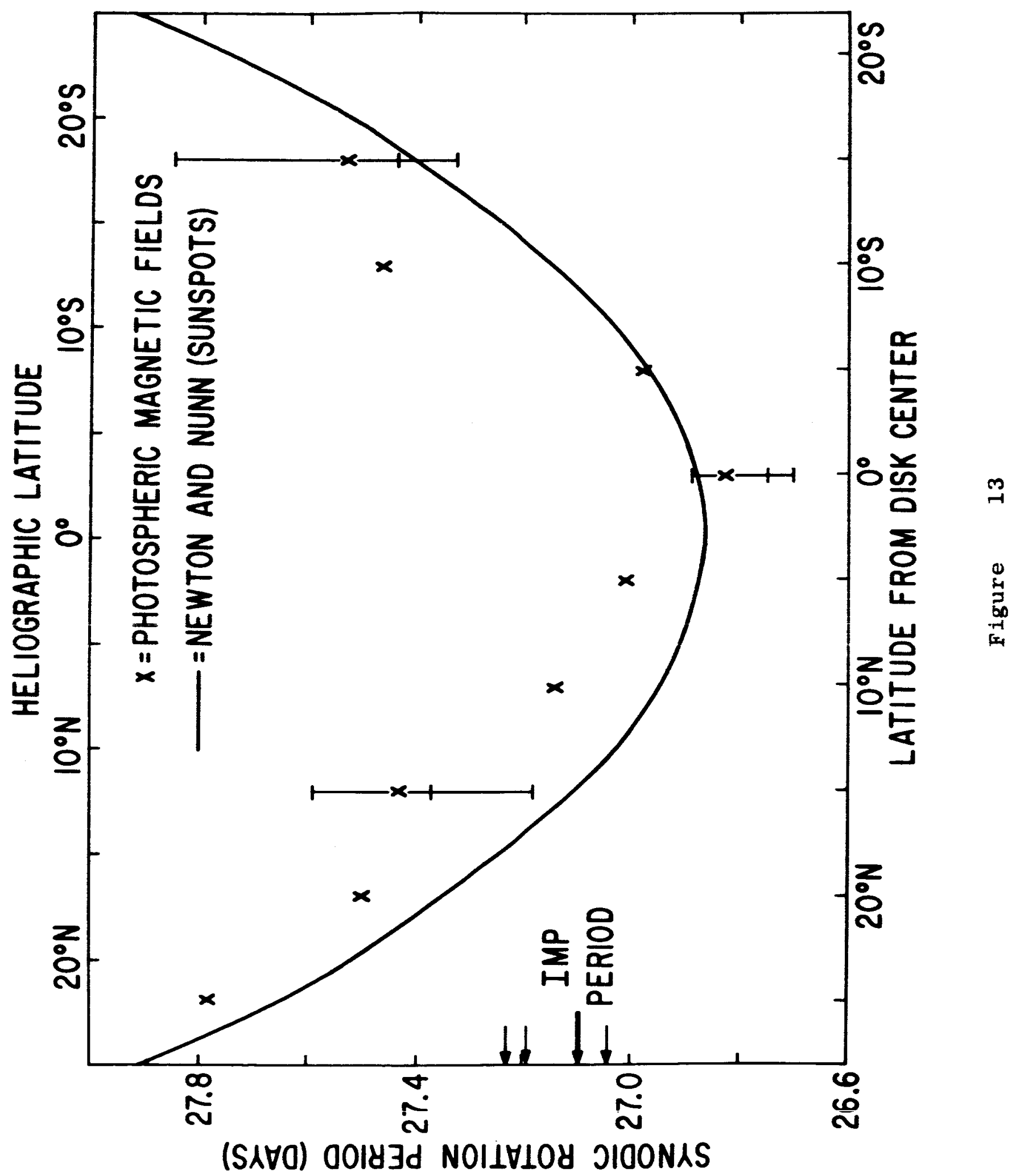




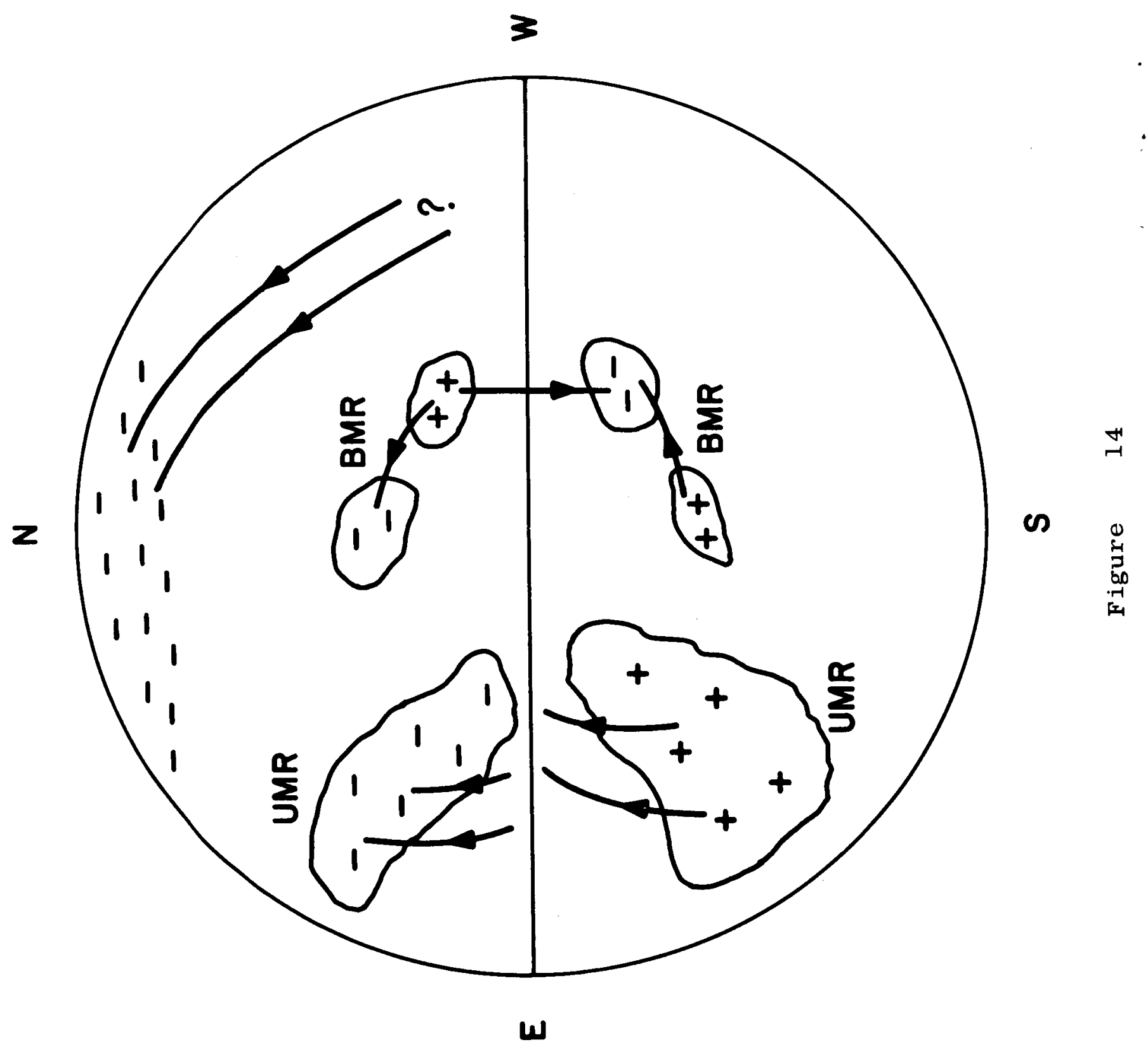

